\title{
Risk factors of pregnancy outcome in patients with pulmonary hypertension
}

\section{Kaixun Zhao}

Guangdong Provincial People's Hospital

\section{Ziyang Yang}

Guangdong Provincial People's Hospital

\section{Yin Zhou}

Guangdong Provincial People's Hospital

\section{Nanshan Xie}

Guangdong Provincial People's Hospital

\section{Disheng Lai}

Guangdong Provincial People's Hospital

Caojin Zhang ( $\sim$ gdzcjpaper@163.com )

Guangdong Provincial People's Hospital

\section{Research Article}

Keywords: Pulmonary hypertension, Pregnancy outcomes, Echocardiography, Prenatal intervention

Posted Date: July 8th, 2022

DOI: https://doi.org/10.21203/rs.3.rs-1234550/v2

License: (c) (1) This work is licensed under a Creative Commons Attribution 4.0 International License.

Read Full License 


\title{
Risk factors of pregnancy outcome in patients with pulmonary hypertension
}

\author{
Kaixun Zhao ${ }^{1}$, Ziyang Yang ${ }^{1}$, Yin Zhou ${ }^{1}$, Nanshan Xie ${ }^{1}$, Disheng Lai ${ }^{1}$, Caojin Zhang ${ }^{1 *}$ \\ ${ }^{1}$ Guangdong Provincial People’s Hospital, Guangdong Academy of Medical Science, \\ Guangdong Institute of Cardiovascular Disease, Guangzhou, GD, CHN
}

Submitting author:

Kaixun Zhao <974111497@qqq.com>

Other authors:

Ziyang Yang <yangziyang93@,hotmail.com>

Yin Zhou <zhouyin199408@163.com>

Nanshan Xie <13417004408@163.com>

Disheng Lai < $\underline{\text { disheng.lai@hotmail.com> }}$

* Corresponding author:

Caojin Zhang <gdzcjpaper@163.com>

Keywords: Pulmonary hypertension, Pregnancy outcomes, Echocardiography, Prenatal intervention 


\title{
Risk factors of pregnancy outcome in patients with pulmonary hypertension
}

\begin{abstract}
OBJECTIVE: The purpose of this study is to explore the factors affecting the pregnancy outcome of patients with pulmonary hypertension, and to design a digital model predicting the pregnancy outcome of patients and judge whether patients have the conditions to continue pregnancy. METHODS: The clinical data of patients with pregnancy complicated with pulmonary hypertension hospitalized in Guangdong Provincial People's Hospital from January 1, 2014 to December 31, 2020 were retrospectively analyzed. The severity of pulmonary hypertension, the structural and functional indexes measured by echocardiography were compared, and the effects on maternal and neonatal outcomes were analyzed. RESULTS: A total of 158 patients met the inclusion and exclusion criteria. The results showed that tricuspid regurgitation velocity $(\mathrm{P}<0.001)$, right ventricular diameter $(\mathrm{P}<0.05)$, right atrial diameter $(\mathrm{P}<0.05)$, pulmonary artery diameter $(\mathrm{P}<0.001)$ and cardiac function classification $(\mathrm{P}<0.001)$ were risk factors for maternal outcomes; Tricuspid regurgitation velocity $(\mathrm{P}<0.001)$ and structural changes of right heart were risk factors for neonatal outcomes. By multivariate logistic regression analysis, tricuspid regurgitation velocity $(\mathrm{P}<0.001)$, cardiac function $(\mathrm{P}<0.05)$ and superior inferior diameter of right atrium $(\mathrm{P}<0.05)$ were significant risk factors for maternal outcomes, while tricuspid regurgitation velocity $(\mathrm{P}<0.001)$ and superior inferior diameter of right atrium $(\mathrm{P}<0.05)$ were significant risk factors for neonatal outcomes. Using significant risk factors, a risk score system was established to predict the pregnancy outcome. CONCLUSION: in this study, we analyzed the clinical data of patients with pregnancy complicated with pulmonary hypertension in our hospital in recent years, and found that the changes of right heart structure (the acceleration of tricuspid regurgitation velocity, and the increase of right atrium, right ventricular diameter, right ventricular outflow tract anteroposterior diameter and main pulmonary artery diameter), are influencing factors of pregnancy outcomes in patients with pulmonary hypertension. In addition, we preliminarily designed a risk score through multi factor analysis, which is helpful to predict the possible outcome of puerperium and neonatal outcomes of patients, and to provide some reference for clinicians and patients to make decisions on whether to
\end{abstract}


continue pregnancy in clinical practice.

\section{Background}

According to the standard definition of pulmonary hypertension $(\mathrm{PH})$ by the World Health Organization (WHO), the hemodynamic diagnostic standard of PH in clinic is generally the mean pulmonary artery pressure (MPAP) $>25 \mathrm{mmhg}$, measured by right cardiac catheterization at sea level and when the patient is at rest. However, in view of the special group of pregnant women, invasive operations such as right cardiac catheterization are not routinely performed clinically, and considering that radiation may have certain potential damage to the fetus, so echocardiography and tricuspid regurgitation are often used to estimate patients' pulmonary systolic pressure in clinic, that is, according to the principle of Bernoulli equation, pulmonary systolic pressure $=4 \times$ Maximum tricuspid regurgitation velocity $2+$ estimated right atrial pressure [1], combined with the diagnostic criteria of tricuspid regurgitation method for the diagnosis of pulmonary hypertension. If the pulmonary systolic pressure is $\geq 35 \mathrm{mmHg}$, pulmonary hypertension can also be initially diagnosed [2].

It is widely believed that pulmonary hypertension is the result of endothelial cell dysfunction, vasoconstriction and vascular remodeling. Common causes of elevated blood volume include left to right shunt caused by congenital heart disease, pregnancy, renal insufficiency and other common causes [3]. At present, it is considered that the increase of pulmonary vascular resistance may be mainly due to the progressive proliferation or resistance to apoptosis of pulmonary artery wall cells, resulting in pulmonary artery wall remodeling [4].

During normal pregnancy, systemic and pulmonary vessels dilate, resulting in decreased systemic (SVR) and pulmonary vascular resistance (PVR). When patients have pulmonary hypertension, pulmonary vascular disease can prevent the decline of PVR, resulting in further increase of pulmonary artery pressure and increase of cardiac output [5]. The effects of various hormones during pregnancy are also involved in the process of hemodynamic changes. Progesterone can also change the tension of smooth muscle, increase vasodilation and volume, and aggravate intravascular stasis [6]. Therefore, during pregnancy, under the action of the above factors and hemodynamic changes, the body presents a hypercoagulable state during pregnancy [7]. Under normal circumstances, the body can adapt to the hemodynamic changes during pregnancy by 
vasodilation and regulating vascular resistance, and generally there will be no obvious cardiopulmonary organ damage or cardiac function involvement.

It is reported that due to such changes in circulation and hemodynamics, the maternal mortality rate is as high as $30-56 \%$. Maternal deaths may occur before, during and after childbirth. Several single center studies at home and abroad have also supported similar conclusions. The mortality of women with idiopathic pulmonary hypertension is $30 \%$, that of patients with congenital heart disease-related pulmonary hypertension is $36 \%$, and that of patients with other diseases such as connective tissue disease-related pulmonary hypertension is about 56\% [8]. According to the statistical analysis of the whole population, it can be roughly speculated that the number of cases per million pregnant women is 178 . Although vascular targeted drugs have been widely used, the mortality is still very high [9].

\section{Research data and methods}

2.1 Study subjects: patients with pregnancy complicated with pulmonary hypertension hospitalized in Guangdong Provincial People's Hospital from January 1, 2014 to December 31, 2020 were studied.

Inclusion criteria: patients who have completed cardiac ultrasound examination and have regular follow-up visits in our hospital or local hospital, and whose pulmonary artery systolic pressure (ePASP) $\geq 35 \mathrm{mmhg}$ estimated by echocardiography before delivery, including class I and class II pulmonary hypertension.

Exclusion criteria: The results of ultrasonography were incomplete, and the tricuspid regurgitation velocity could not be measured.

2.2 Endpoints of research: two endpoints were set in this study, including maternal outcome and neonatal outcome. The end point of maternal outcome was that the maternal was transferred to $\mathrm{CCU}$ for further monitoring and treatment. The end point of newborns was full-term, premature or stillbirth.

2.3 Test method: all patients were placed in the left decubitus position and tested with ACUSON Sequoia c256 (ACUSON, mountain view, CA, USA) or Ge Vivid 7 echocardiography 
diagnostic instrument.

2.4 Observation indexes: age, gestational week, laboratory examination, pregnancy outcome, risk factors related to pulmonary vascular disease (chronic kidney disease, blood system disease, use history of targeted drugs, infection history of special pathogens, etc.), and relevant parameters of echocardiography (including tricuspid regurgitation velocity, E / a ratio of cardiac function index and LVEF, Cardiac structural indexes (left atrial diameter, left ventricular end diastolic diameter, left ventricular end systolic diameter, right atrial upper and lower diameter, right ventricular upper and lower diameter, main pulmonary artery diameter, etc.).

Considering that the patient is pregnant and parturient, it is not suitable to use right heart catheterization, but the error of ultrasonic estimation of pulmonary artery systolic pressure is large. Therefore, in this study, tricuspid regurgitation velocity is used as an index to estimate right ventricular systolic pressure and assess the severity of pulmonary hypertension. In addition, the classification variables such as cardiac function classification and pulmonary hypertension classification were also included in the observation indexes.

2.5 Statistical methods: SPSS 25.0 statistical software (USA ill. IBM) was used for data analysis. The rank sum test of parameters was used for the distribution difference of grade data, the measurement data was expressed by mean \pm standard deviation, and the chi square test and t-test were used for independent sample data; The risk factors are screened by single factor Logistic binary regression analysis. In order to prevent the omission of important variables, we adjust the screening standard to $\mathrm{P} \leq 0.10$, conduct logistic multi factor regression analysis on the significant factors, and establish the scoring model according to the multi factor regression analysis. For the groups with small samples, descriptive analysis and statistical chart are used. When $\mathrm{P}<0.05$, it is considered that the difference is statistically significant.

\section{Result}

3.1.1 Analysis of risk factors affecting maternal outcome

There were 158 patients whose clinical baseline data met the criteria, including 94 patients with pulmonary arterial hypertension, including 46 patients with congenital heart disease, 17 
patients with Eisenmenger syndrome, 31 patients with idiopathic pulmonary hypertension and 64 patients with left heart disease related pulmonary hypertension. According to the maternal outcome, the patients were divided into two groups: transferred to cardiac care unit (CCU) and non-transferred to CCU. According to the results of T-test, There were significant differences in red blood cells (3.97 \pm 0.51 vs $4.31 \pm 0.79, \mathrm{P}=0.002)$, Hemoglobin concentration (113.28 \pm 16.15 vs $122.98 \pm 21.43$, $\mathrm{P}=0.002)$, B-type natriuretic peptide precursor ( $292.31 \pm 673.92$ vs $635.42 \pm 742.47, \mathrm{P}=0.006$ ), Fibrin $(4.83 \pm 2.28$ vs $4.21 \pm 0.94, \mathrm{p}=0.017)$, Tricuspid regurgitation velocity $(299.43 \pm 42.62$ vs $416.18 \pm 81.29, \mathrm{P}<0.0001)$, upper and lower diameter of right atrium (49.96 \pm 8.90 vs $54.43 \pm 8.92$, $\mathrm{P}=0.009)$, upper and lower diameter of right ventricle (54.68 \pm 7.66 vs $59.16 \pm 8.92, \mathrm{P}=0.005)$ and the diameter of main pulmonary artery $(26.39 \pm 4.88$ vs $32.52 \pm 7.80, \mathrm{P}<0.0001)$. There were also significant differences in classification of pulmonary hypertension and cardiac function. See Table 1-1 and 1-2 for the comparison between other groups.

Table 1-1. Maternal baseline data (maternal outcomes)

\begin{tabular}{|c|c|c|c|}
\hline & non-transferred to $\mathrm{CCU} \quad(\mathrm{n}=114)$ & transferred to $\mathrm{CCU} \quad(\mathrm{n}=44)$ & $P$ value \\
\hline Age (years) & $31.54( \pm 5.08)$ & $30.52( \pm 4.96)$ & 0.253 \\
\hline WBC & $9.35( \pm 2.46)$ & $9.59( \pm 3.00)$ & 0.637 \\
\hline $\mathrm{RBC}$ & $3.97( \pm 0.51)$ & $4.31( \pm 0.79)$ & 0.002 \\
\hline HGB & $113.28( \pm 16.15)$ & $122.98( \pm 21.43)$ & 0.002 \\
\hline PLT & $223.81( \pm 63.35)$ & $185.73( \pm 55.47)$ & 0.000 \\
\hline HCT & $0.34( \pm 0.04)$ & $0.37( \pm 0.06)$ & 0.000 \\
\hline $\mathrm{CK}$ & $87.20( \pm 141.22)$ & $65.42( \pm 52.24)$ & 0.159 \\
\hline CK-MB & $12.17( \pm 6.64)$ & $14.36( \pm 13.32)$ & 0.171 \\
\hline LDH & $220.07( \pm 95.14)$ & $227.95( \pm 51.83)$ & 0.543 \\
\hline $\mathrm{HBDH}$ & $151.15( \pm 56.68)$ & $159.02( \pm 45.39)$ & 0.375 \\
\hline ProBNP & $292.31( \pm 673.92)$ & $635.42( \pm 742.47)$ & 0.006 \\
\hline TNT-HS & $9.37( \pm 15.86)$ & $8.89( \pm 5.94)$ & 0.780 \\
\hline INR & $1.16( \pm 0.74)$ & $1.03( \pm 0.14)$ & 0.240 \\
\hline PT-A & $97.74( \pm 23.53)$ & $99.00( \pm 15.62)$ & 0.697 \\
\hline PT & $15.77( \pm 13.68)$ & $13.67( \pm 1.20)$ & 0.302 \\
\hline APTT & $34.47( \pm 7.55)$ & $35.15( \pm 3.32)$ & 0.568 \\
\hline FIB & $4.83( \pm 2.28)$ & $4.21( \pm 0.94)$ & 0.017 \\
\hline TT & $15.64( \pm 3.69)$ & $15.29( \pm 1.36)$ & 0.392 \\
\hline DDI & $1503.42( \pm 1364.14)$ & $1424.77( \pm 1196.40)$ & 0.723 \\
\hline \multicolumn{4}{|l|}{ Cardiac Function } \\
\hline I-II $(\%)$ & $108(94.73)$ & $24(54.55)$ & 0.000 \\
\hline III-IV（\%） & $6(5.27)$ & $20(45.45)$ & \\
\hline \multicolumn{4}{|l|}{ Etiology of PH } \\
\hline Congenital heart disease $(\%)$ & $35(30.70)$ & $28(63.64)$ & 0.001 \\
\hline Idiopathic pulmonary hypertension (\%) & $26(22.81)$ & $5(11.36)$ & \\
\hline Left heart disease $(\%)$ & $53(46.49)$ & $11(25.00)$ & \\
\hline
\end{tabular}

Values are $\mathrm{n}(\%)$ or mean \pm standard deviation.

WBC, white blood cells; RBC, red blood cells; HGB, hemoglobin; PLT, platelet; HCT, hematocrit; CK, creatine kinase; CK-MB, Creatine phosphokinase isoenzyme; LDH, lactate dehydrogenase; HBDH, Hydroxybutyrate dehydrogenase; ProBNP, Brain natriuretic peptide precursor; TNT-HS, high sensitivity troponin T; INR, international normalized ratio; PT-A, prothrombin activity; PT, prothrombin time; APTT, activated partial thrombin time; FIB, fibrin; TT, thrombin time; PH, pulmonary hypertension. 
Table 1-2. Maternal echocardiographic results (maternal outcome)

\begin{tabular}{|c|c|c|c|}
\hline & non-transferred to $\mathrm{CCU} \quad(\mathrm{n}=114)$ & transferred to $\mathrm{CCU} \quad(\mathrm{n}=44)$ & $P$ value \\
\hline \multicolumn{4}{|l|}{ Maternal echocardiographic data } \\
\hline $\mathrm{E}(\mathrm{m} / \mathrm{s})$ & $1.36( \pm 0.64)$ & $1.14( \pm 0.74)$ & 0.089 \\
\hline $\mathrm{A}(\mathrm{m} / \mathrm{s})$ & $1.08( \pm 0.62)$ & $0.91( \pm 0.64)$ & 0.131 \\
\hline $\mathrm{E} / \mathrm{A}$ & $1.39( \pm 0.49)$ & $1.37( \pm 0.53)$ & 0.786 \\
\hline $\operatorname{LVEF}(\%)$ & $64.38( \pm 5.70)$ & $65.57( \pm 10.13)$ & 0.353 \\
\hline TI $(\mathrm{cm} / \mathrm{s})$ & $299.43( \pm 42.62)$ & $416.18( \pm 81.29)$ & 0.000 \\
\hline $\mathrm{RA}(\mathrm{mm})$ & $40.05( \pm 7.54)$ & $39.18( \pm 9.69)$ & 0.550 \\
\hline Anteroposterior diameter of RVOT $(\mathrm{mm})$ & $25.83( \pm 6.53)$ & $27.16( \pm 7.84)$ & 0.323 \\
\hline LV end diastolic diameter $(\mathrm{mm})$ & $45.93( \pm 6.02)$ & $44.84( \pm 8.50)$ & 0.368 \\
\hline LV end systolic diameter $(\mathrm{mm})$ & $28.83( \pm 5.48)$ & $28.16( \pm 7.79)$ & 0.541 \\
\hline RA superior and inferior diameter $(\mathrm{mm})$ & $49.96( \pm 8.90)$ & $54.43( \pm 9.58)$ & 0.009 \\
\hline $\mathrm{RV}$ superior and inferior diameter $(\mathrm{mm})$ & $54.68( \pm 7.66)$ & $59.16( \pm 8.92)$ & 0.005 \\
\hline Main pulmonary artery $(\mathrm{mm})$ & $26.39( \pm 4.88)$ & $32.52( \pm 7.80)$ & 0.000 \\
\hline Ventricular septal thickness（mm） & $9.01( \pm 1.78)$ & $9.50( \pm 1.96)$ & 0.152 \\
\hline LV posterior wall thickness $(\mathrm{mm})$ & $8.81( \pm 1.30)$ & $9.11( \pm 1.10)$ & 0.152 \\
\hline
\end{tabular}

Values are $\mathrm{n}(\%)$ or mean \pm standard deviation.

E, Maximum early diastolic velocity of mitral valve; A, Maximum velocity of early mitral systole; LVEF, left ventricular ejection fraction; TI, tricuspid regurgitation velocity; RA, right atrium; RV, right ventricle; RVOT, right ventricular outflow tract; LV, left ventricle.

\subsubsection{Univariate and multivariate analysis of maternal outcomes}

Univariate analysis of maternal outcome: through preliminary screening, there were significant differences in erythrocyte count, hemoglobin concentration, cardiac function, clinical classification of pulmonary hypertension, tricuspid regurgitation velocity, upper and lower diameter of right atrium, upper and lower diameter of right ventricle and main pulmonary artery diameter between the two groups. The significant variables were first analyzed by univariate logistic regression. According to the diagnostic guidelines of adult right heart echocardiography of American ultrasonic Association (ASE) and the standards recommended in the measurement guidelines of Chinese adult echocardiography by the echocardiography group of ultrasonic medical branch of Chinese Medical Association, various continuous indexes were analyzed in the form of grade data, including tricuspid regurgitation velocity, upper and lower diameter of right atrium, upper and lower diameter of right ventricle, diameter of main pulmonary artery The classification of cardiac function was a significant single factor of maternal outcome $(\mathrm{P}<0.05)$. The detailed results are shown in table 1-3.

According to the results of logistic univariate regression analysis, the significant univariate 
regression analysis showed that tricuspid regurgitation velocity $\leq 3.4 \mathrm{~m} / \mathrm{s}(\mathrm{b}=-2.776, \mathrm{P}<0.001)$, upper and lower diameter of right atrium $\leq 50 \mathrm{~mm}(\mathrm{~b}=-1.396, \mathrm{P}<0.05)$ and cardiac function grade $\mathrm{I}-\mathrm{II}(\mathrm{b}=-$ 1.888, $\mathrm{P}<0.05$ ) had significant negative effects on maternal transfer to $\mathrm{CCU}$, The upper and lower diameter of right ventricle and the diameter of main pulmonary artery had no significant effect on maternal outcome.

Table 1-3. Univariate and multivariate analysis of maternal outcomes

\begin{tabular}{|c|c|c|c|c|c|c|c|c|c|}
\hline \multirow[b]{3}{*}{ TI } & \multirow[b]{3}{*}{$\leqslant 3.4$} & \multicolumn{4}{|c|}{ Univariate analysis } & \multicolumn{4}{|c|}{ Multivariate analysis } \\
\hline & & \multirow{2}{*}{$\begin{array}{c}\text { B } \\
-2.793\end{array}$} & \multicolumn{2}{|c|}{$95 \%$ CI } & \multirow{2}{*}{$\begin{array}{c}\mathrm{P} \\
<0.001\end{array}$} & \multirow{2}{*}{$\begin{array}{c}\text { B } \\
-2.776\end{array}$} & \multicolumn{2}{|c|}{$95 \% \mathrm{CI}$} & \multirow{2}{*}{$\begin{array}{c}\mathrm{P} \\
<0.001\end{array}$} \\
\hline & & & -3.641 & -1.945 & & & -3.927 & -1.626 & \\
\hline & $>3.4$ & & & & & & & & \\
\hline \multirow[t]{2}{*}{ RA superior and inferior diameter } & $\leqslant 50 \mathrm{~mm}$ & -1.274 & -2.007 & -0.542 & $<0.05$ & -1.396 & -2.507 & -0.284 & $<0.05$ \\
\hline & $>50 \mathrm{~mm}$ & & & & & & & & \\
\hline \multirow[t]{2}{*}{ RV superior and inferior diameter } & $\leqslant 65 \mathrm{~mm}$ & -1.485 & -2.476 & -0.494 & $<0.05$ & & & & \\
\hline & $>65 \mathrm{~mm}$ & & & & & & & & \\
\hline \multirow[t]{2}{*}{ Main pulmonary artery } & $\leqslant 27 \mathrm{~mm}$ & -1.455 & -2.2 & -0.711 & $<0.001$ & & & & \\
\hline & $>27 \mathrm{~mm}$ & & & & & & & & \\
\hline \multirow[t]{3}{*}{ RBC } & $<3.5 \times 10 \mathrm{e} 12 / \mathrm{L}$ & -1.269 & -2.683 & 0.146 & 0.079 & & & & \\
\hline & $3.5-5.0 \times 10 \mathrm{e} 12 / \mathrm{L}$ & -1.408 & -2.544 & -0.272 & $<0.05$ & & & & \\
\hline & $>5.0 \times 10 \mathrm{e} 12 / \mathrm{L}$ & & & & & & & & \\
\hline \multirow[t]{3}{*}{ HGB } & $\geqslant 110 \mathrm{~g} / \mathrm{L}$ & 0.62 & -0.983 & 2.224 & 0.448 & & & & \\
\hline & $90-109 \mathrm{~g} / \mathrm{L}$ & 0.028 & -1.686 & 1.742 & 0.974 & & & & \\
\hline & $60-89 \mathrm{~g} / \mathrm{L}$ & & & & & & & & \\
\hline \multirow[t]{2}{*}{ Cardiac function } & I-II & -2.708 & -3.722 & -1.694 & $<0.001$ & -1.888 & -3.205 & -0.571 & $<0.05$ \\
\hline & III-IV & & & & & & & & \\
\hline \multirow[t]{2}{*}{ Classification } & $\mathrm{PAH}$ & 0.958 & 0.183 & 1.733 & $<0.05$ & & & & \\
\hline & PH & & & & & & & & \\
\hline
\end{tabular}

\subsubsection{Risk score of pregnancy outcome}

In the total population, according to the results of multivariate logistic regression analysis, the effects of tricuspid regurgitation velocity, upper and lower diameter of right atrium and cardiac function on adverse pregnancy outcome are significant. A scoring system for predicting pregnancy outcome was established based on tricuspid regurgitation velocity, right atrial upper and lower diameter and cardiac function classification. In the model for predicting maternal outcome, the tricuspid regurgitation velocity $\leq 3.4 \mathrm{~m} / \mathrm{s}$ is 0 points, $>3.4 \mathrm{~m} / \mathrm{s}$ is 5 points. The upper and lower diameter of the right atrium $\leq 50 \mathrm{~mm}$ is 2 points, greater than $50 \mathrm{~mm}$ is 5 points. The cardiac function I-II is 1 point, III-IV is 5 points, and the total score ranges from 3 to 15 points. The detailed results 
are shown in table 1-4 for specific scores.

Table 1-4. Risk scores for predicting maternal outcomes

\begin{tabular}{cccc}
\hline Risk factors & Grouping & regression coefficient & Score \\
\hline $\begin{array}{c}\text { Tricuspid regurgitation } \\
\text { velocity }\end{array}$ & $\leqslant 3.40 \mathrm{~m} / \mathrm{s}$ & -2.776 & 0 \\
& $>3.40 \mathrm{~m} / \mathrm{s}$ & & 5 \\
$\begin{array}{c}\text { RA superior and inferior } \\
\text { diameter }\end{array}$ & $\leqslant 50 \mathrm{~mm}$ & -1.396 & 2 \\
& $>50 \mathrm{~mm}$ & & 5 \\
Cardiac function & I-II & -1.888 & 1 \\
& III-IV & & 5 \\
\hline
\end{tabular}

Pearson chi square test was used to test the scoring system. The results showed that the score had significant statistical significance in predicting neonatal outcome $(\mathrm{P}<0.001)$. In addition, the receiver operating characteristic (ROC) curve was used to predict the best cut-off value of score. The study found that the best cut-off value of score for predicting maternal low-risk outcome was 7 points, $\mathrm{AUC}=0.886, \mathrm{P}<0.001$ (Table 1-1); Therefore, when the patient risk score is less than 7 points, the patient is at low risk. In addition, patients with scores greater than 7 points were analyzed. There were differences between different scores. The best score boundary for predicting maternal highrisk outcomes was 10.5 points, $\mathrm{AUC}=0.794, \mathrm{P}<0.05$ (Table 1-2). Therefore, the score of 8-10 points is medium risk and larger than 11 points is high risk. See table 1-5 for detailed scoring criteria. 
Table 1-5. Risk score and maternal outcomes

\begin{tabular}{|c|c|c|}
\hline Risk factors & Score & Risk \\
\hline $\mathrm{TI} \leqslant 3.4 \mathrm{~m} / \mathrm{s}, \quad \mathrm{RA} \leqslant 50 \mathrm{~mm}, \quad$ NYHA I-II & 3 & \multirow[t]{3}{*}{ Low } \\
\hline $\mathrm{TI} \leqslant 3.4 \mathrm{~m} / \mathrm{s}, \quad$ RA $>50 \mathrm{~mm}, \quad$ NYHA I-II & 6 & \\
\hline $\mathrm{TI} \leqslant 3.4 \mathrm{~m} / \mathrm{s}, \quad \mathrm{RA} \leqslant 50 \mathrm{~mm}, \quad$ NYHA III-IV & 7 & \\
\hline $\mathrm{TI}>3.4 \mathrm{~m} / \mathrm{s}, \quad \mathrm{RA} \leqslant 50 \mathrm{~mm}, \quad$ NYHA I-II & 8 & \multirow[t]{2}{*}{ Medium } \\
\hline $\mathrm{TI} \leqslant 3.4 \mathrm{~m} / \mathrm{s}, \quad \mathrm{RA}>50 \mathrm{~mm}, \quad$ NYHA III-IV & 10 & \\
\hline $\mathrm{TI}>3.4 \mathrm{~m} / \mathrm{s}, \quad \mathrm{RA}>50 \mathrm{~mm}, \quad$ NYHA I-II & 11 & \multirow{3}{*}{ High } \\
\hline $\mathrm{TI}>3.4 \mathrm{~m} / \mathrm{s}, \quad \mathrm{RA} \leqslant 50 \mathrm{~mm}, \quad$ NYHA III-IV & 12 & \\
\hline $\mathrm{TI}>3.4 \mathrm{~m} / \mathrm{s}, \quad \mathrm{RA}>50 \mathrm{~mm}, \quad$ NYHA III-IV & 15 & \\
\hline
\end{tabular}

\subsection{Analysis of risk factors affecting neonatal outcomes}

3.2.1 According to the results of t-test, there were significant differences in Hemoglobin concentration $(112.74 \pm 13.49$ vs $119.39 \pm 21.74, \mathrm{P}=0.021)$, tricuspid regurgitation velocity $(299.49 \pm 51.90$ vs $366.08 \pm 83.64, \mathrm{P}<0.0001)$ and upper and lower diameter of right atrium (49.49 \pm 8.54 vs $53.01 \pm 9.74, \mathrm{P}=0.017$ ), anterior posterior diameter of right ventricular outflow tract ( $25.04 \pm 6.50$ vs $27.43 \pm 7.17, \mathrm{P}=0.030$ ), main pulmonary artery diameter $(26.38 \pm 5.33$ vs $29.91 \pm 7.02$, $\mathrm{P}<0.0001)$. There were also significant differences in the classification of cardiac function and pulmonary hypertension. See table 2-1 and 2-2 for the comparison between other groups. 
Table 2-1. Maternal baseline data (neonatal outcomes)

\begin{tabular}{|c|c|c|c|}
\hline & Full term $(\mathrm{n}=81)$ & Premature birth + stillbirth $(\mathrm{n}=77)$ & $P$ value \\
\hline Age (years) & $31.37( \pm 5.19)$ & $31.14( \pm 4.95)$ & 0.205 \\
\hline WBC & $9.16( \pm 2.09)$ & $9.69( \pm 3.07)$ & 0.891 \\
\hline $\mathrm{RBC}$ & $3.99( \pm 0.41)$ & $4.14( \pm 0.78)$ & 0.139 \\
\hline HGB & $112.74( \pm 13.49)$ & $119.39( \pm 21.74)$ & 0.021 \\
\hline PLT & $219.75( \pm 58.55)$ & $206.31( \pm 67.91)$ & 0.186 \\
\hline HCT & $0.34( \pm 0.04)$ & $0.36( \pm 0.06)$ & 0.009 \\
\hline CK & $108.00( \pm 165.46)$ & $52.88( \pm 31.63)$ & 0.005 \\
\hline CK-MB & $12.67( \pm 7.17)$ & $12.90( \pm 10.66)$ & 0.877 \\
\hline LDH & $225.07( \pm 104.80)$ & $218.94( \pm 58.46)$ & 0.648 \\
\hline ProBNP & $170.87( \pm 177.11)$ & $616.12( \pm 948.87)$ & 0.000 \\
\hline TNT-HS & $7.94( \pm 11.05)$ & $10.60( \pm 16.18)$ & 0.233 \\
\hline INR & $1.07( \pm 0.47)$ & $1.18( \pm 0.77)$ & 0.279 \\
\hline PT-A & $101.53( \pm 20.90)$ & $94.47( \pm 21.82)$ & 0.040 \\
\hline PT & $14.68( \pm 9.14)$ & $15.72( \pm 13.87)$ & 0.578 \\
\hline APTT & $33.68( \pm 5.06)$ & $33.68( \pm 7.89)$ & 0.062 \\
\hline FIB & $4.71( \pm 1.25)$ & $4.60( \pm 2.60)$ & 0.723 \\
\hline TT & $15.14( \pm 2.51)$ & $15.96( \pm 3.79)$ & 0.115 \\
\hline DDI & $1634.32( \pm 1473.53)$ & $1320.78( \pm 1114.75)$ & 0.132 \\
\hline \multicolumn{4}{|l|}{ Cardiac Function } \\
\hline I-II $(\%)$ & $77(95.06)$ & $54(70.13)$ & 0.000 \\
\hline III-IV (\%) & $4(4.94)$ & $23(29.87)$ & \\
\hline \multicolumn{4}{|l|}{ Etiology of PH } \\
\hline Congenital heart disease $(\%)$ & $31(38.27)$ & $32(41.56)$ & 0.134 \\
\hline Idiopathic pulmonary hypertension $(\%)$ & $10(12.35)$ & $21(27.27)$ & \\
\hline Left heart disease $(\%)$ & $40(49.38)$ & $24(31.17)$ & \\
\hline
\end{tabular}

Values are $\mathrm{n}(\%)$ or mean \pm standard deviation.

WBC, white blood cells; RBC, red blood cells; HGB, hemoglobin; PLT, platelet; HCT, hematocrit; CK, creatine kinase; CK-MB, Creatine phosphokinase isoenzyme; LDH, lactate dehydrogenase; HBDH, Hydroxybutyrate dehydrogenase; ProBNP, Brain natriuretic peptide precursor; TNT-HS, high sensitivity troponin T; INR, international normalized ratio; PT-A, prothrombin activity; PT, prothrombin time; APTT, activated partial thrombin time; FIB, fibrin; TT, thrombin time; PH, pulmonary hypertension.

Table 2-2. Maternal echocardiographic results (neonatal outcomes)

\begin{tabular}{|c|c|c|c|}
\hline & Full term $(\mathrm{n}=81)$ & Premature birth + stillbirth $(\mathrm{n}=77)$ & $P$ value \\
\hline \multicolumn{4}{|l|}{ Maternal echocardiographic data } \\
\hline $\mathrm{E}(\mathrm{m} / \mathrm{s})$ & $1.40( \pm 0.65)$ & $1.20( \pm 0.68)$ & 0.065 \\
\hline $\mathrm{A}(\mathrm{m} / \mathrm{s})$ & $1.11( \pm 0.65)$ & $0.94( \pm 0.60)$ & 0.092 \\
\hline $\mathrm{E} / \mathrm{A}$ & $1.40( \pm 0.45)$ & $1.39( \pm 0.55)$ & 0.914 \\
\hline $\operatorname{LVEF}(\%)$ & $65.23( \pm 5.54)$ & $64.15( \pm 8.60)$ & 0.348 \\
\hline $\mathrm{TI}(\mathrm{cm} / \mathrm{s})$ & $299.49( \pm 51.90)$ & $366.08( \pm 83.64)$ & 0.000 \\
\hline $\mathrm{RA}(\mathrm{mm})$ & $40.16( \pm 6.53)$ & $39.44( \pm 9.63)$ & 0.582 \\
\hline Anteroposterior diameter of RVOT ( $\mathrm{mm}$ ) & $25.04( \pm 6.50)$ & $27.43( \pm 7.17)$ & 0.030 \\
\hline LV end diastolic diameter $(\mathrm{mm})$ & $46.36( \pm 5.99)$ & $44.86( \pm 7.51)$ & 0.168 \\
\hline LV end systolic diameter $(\mathrm{mm})$ & $29.23( \pm 5.05)$ & $28.03( \pm 7.18)$ & 0.221 \\
\hline RA superior and inferior diameter $(\mathrm{mm})$ & $49.49( \pm 8.54)$ & $53.01( \pm 9.74)$ & 0.017 \\
\hline $\mathrm{RV}$ superior and inferior diameter $(\mathrm{mm})$ & $54.70( \pm 7.40)$ & $57.22( \pm 8.92)$ & 0.056 \\
\hline Main pulmonary artery $(\mathrm{mm})$ & $26.38( \pm 5.33)$ & $29.91( \pm 7.02)$ & 0.000 \\
\hline Ventricular septal thickness $(\mathrm{mm})$ & $9.01( \pm 1.43)$ & $9.29( \pm 2.19)$ & 0.358 \\
\hline LV posterior wall thickness $(\mathrm{mm})$ & $8.88( \pm 1.25)$ & $8.91( \pm 1.27)$ & 0.871 \\
\hline
\end{tabular}

Values are $\mathrm{n}(\%)$ or mean \pm standard deviation.

E, Maximum early diastolic velocity of mitral valve; A, Maximum velocity of early mitral systole; LVEF, left ventricular ejection fraction; 


\subsubsection{Univariate and multivariate analysis of neonatal outcomes}

Univariate analysis of neonatal outcome: the variables were screened by variance test and Ttest, and the significant variables were first analyzed by logistic univariate regression. It was found that tricuspid regurgitation velocity, anterior and posterior diameter of right ventricular outflow tract, superior and inferior diameter of right atrium, diameter of main pulmonary artery, classification of cardiac function and classification of pulmonary hypertension were all univariate factors that had a significant impact on neonatal outcome $(\mathrm{P}<0.05)$, Details has shown in table 2-3.

In order to further explore the impact of various variables on neonatal outcomes, the variables passed through univariate analysis were included in multivariate regression for analysis. The results showed that tricuspid regurgitation velocity $\leq 3.4 \mathrm{~m} / \mathrm{s}$ had a significant negative effect on neonatal outcome $(b=-1.234, P<0.05)$, upper and lower diameter of right atrium $\leq 50 \mathrm{~mm}$ also had a significant negative effect on neonatal outcome $(\mathrm{b}=-0.886, \mathrm{P}<0.05)$, anterior and posterior diameter of right ventricular outflow tract, diameter of main pulmonary artery, hemoglobin concentration. And the cardiac function and the type of pulmonary hypertension had no significant effect on neonatal outcome. 
Table 2-3. Univariate and multivariate analysis of neonatal outcomes

\begin{tabular}{|c|c|c|c|c|c|c|c|c|c|}
\hline \multirow[b]{3}{*}{ TI } & \multirow[b]{3}{*}{$\leqslant 3.4$} & \multicolumn{4}{|c|}{ Univariate analysis } & \multicolumn{4}{|c|}{ Multivariate analysis } \\
\hline & & \multirow{2}{*}{$\frac{\mathrm{B}}{-1.619}$} & \multicolumn{2}{|c|}{$95 \% \mathrm{CI}$} & \multirow{2}{*}{$\begin{array}{c}\mathrm{P} \\
<0.001\end{array}$} & \multirow{2}{*}{$\begin{array}{c}\text { B } \\
-1.234\end{array}$} & \multicolumn{2}{|c|}{$95 \% \mathrm{CI}$} & \multirow{2}{*}{$\begin{array}{c}\mathrm{P} \\
<0.05\end{array}$} \\
\hline & & & -2.378 & -0.86 & & & -2.117 & -0.352 & \\
\hline & $>3.4$ & & & & & & & & \\
\hline \multirow[t]{2}{*}{ Anteroposterior diameter of RVOT } & $\leqslant 29.8 \mathrm{~mm}$ & -0.822 & -1.528 & -0.116 & $<0.05$ & & & & \\
\hline & $>29.8 \mathrm{~mm}$ & & & & & & & & \\
\hline \multirow[t]{2}{*}{ RA superior and inferior diameter } & $\leqslant 50 \mathrm{~mm}$ & -1.094 & -1.747 & -0.441 & $<0.05$ & -0.886 & -1.669 & -0.103 & $<0.05$ \\
\hline & $>50 \mathrm{~mm}$ & & & & & & & & \\
\hline \multirow[t]{2}{*}{ RV superior and inferior diameter } & $\leqslant 65 \mathrm{~mm}$ & -0.932 & -1.955 & 0.091 & 0.074 & & & & \\
\hline & $>65 \mathrm{~mm}$ & & & & & & & & \\
\hline \multirow[t]{2}{*}{ Main pulmonary artery } & $\leqslant 27 \mathrm{~mm}$ & -1.041 & -1.693 & -0.39 & $<0.05$ & & & & \\
\hline & $>27 \mathrm{~mm}$ & & & & & & & & \\
\hline \multirow[t]{3}{*}{ HGB } & $\geqslant 110 \mathrm{~g} / \mathrm{L}$ & -0.038 & -1.336 & 1.259 & 0.954 & & & & \\
\hline & $90-109 \mathrm{~g} / \mathrm{L}$ & -0.091 & -1.464 & 1.283 & 0.897 & & & & \\
\hline & $60-89 \mathrm{~g} / \mathrm{L}$ & & & & & & & & \\
\hline \multirow[t]{2}{*}{ Cardiac function } & I-II & -2.041 & -3.161 & -0.921 & $<0.001$ & & & & \\
\hline & III-IV & & & & & & & & \\
\hline \multirow[t]{2}{*}{ Classification } & PAH & 0.768 & 0.118 & 1.417 & $<0.05$ & & & & \\
\hline & PH & & & & & & & & \\
\hline
\end{tabular}

3.2.3 Scoring model for predicting neonatal outcome

According to the results of univariate and multivariate regression analysis, the tricuspid regurgitation velocity $\leq 3.4 \mathrm{~m} / \mathrm{s}$ is 0 and $>3.4 \mathrm{~m} / \mathrm{s}$ is 3 points; The upper and lower diameter of the right atrium $\leq 50 \mathrm{~mm}$ is 1 point, greater than $50 \mathrm{~mm}$ is 3 points, and the total score ranges from 0 to 6 points. See table $2-4$ for detailed scores.

Table 2-4. Risk scores for predicting neonatal outcomes

\begin{tabular}{cccc}
\hline Risk factors & Grouping & regression coefficient & Score \\
\hline $\begin{array}{c}\text { Tricuspid regurgitation } \\
\text { velocity }\end{array}$ & $\leqslant 3.40 \mathrm{~m} / \mathrm{s}$ & -1.234 & 0 \\
& $>3.40 \mathrm{~m} / \mathrm{s}$ & & 3 \\
$\begin{array}{c}\text { RA superior and inferior } \\
\text { diameter }\end{array}$ & $\leqslant 50 \mathrm{~mm}$ & -0.886 & 1 \\
& $>50 \mathrm{~mm}$ & & 3 \\
\hline
\end{tabular}

According to Pearson chi square, the score model was tested. The results showed that the score 
had significant statistical significance in predicting neonatal outcome $(\mathrm{P}<0.001)$. After the receiver operating characteristic (ROC) curve is used to predict the optimal score boundary value, it is suggested that the optimal score boundary value affecting the outcome of low-risk newborns is 1 point, $\mathrm{AUC}=0.736, \mathrm{P}<0.001$ (table 2-1). Therefore, patient is at low risk when the patient risk score is less than 1 point. In addition, it is found that there are differences between different scores in patients with more than 1 point. The best score limit for predicting the outcome of high-risk newborns is 3.5, AUC $=0.634, \mathrm{P}<0.05$ (table 2-2). Therefore, when the risk score is larger than 3 points, the neonatal outcome may be high risk, and when the risk score is between 2 and 3 , it may be medium risk. See table 2-5 for detailed scoring criteria.

Table 2-5. Risk score and neonatal outcomes

\begin{tabular}{|l|c|c|}
\hline \multicolumn{1}{|c|}{ Risk factors } & Score & Risk \\
\cline { 1 - 2 } $\mathbf{T I} \leqslant \mathbf{3 . 4 m} / \mathbf{s}, \quad$ RA $\leqslant \mathbf{5 0 m m}$ & 1 & Low \\
\hline $\mathbf{T I} \leqslant \mathbf{3 . 4 m} / \mathbf{s}, \quad$ RA $>\mathbf{5 0 m m}$ & 3 & Medium \\
\hline $\mathbf{T I}>\mathbf{3 . 4 m} / \mathbf{s}, \quad$ RA $\leqslant \mathbf{5 0 m m}$ & 4 & High \\
\cline { 1 - 2 } $\mathbf{T I}>\mathbf{3 . 4 m} / \mathbf{s}, \quad$ RA $>\mathbf{5 0 m m}$ & 6 & \\
\hline
\end{tabular}

\section{Disscussion}

4.1 Risk factors affecting maternal outcomes

In this study, through data analysis, it was found that in univariate analysis, tricuspid regurgitation velocity, right ventricular diameter, right atrial diameter, pulmonary artery diameter and other right cardiac function and structural indexes, as well as cardiac function, were significant risk factors affecting maternal outcome. However, through multivariate analysis, only tricuspid regurgitation velocity, cardiac function grade and right atrial upper and lower diameter were significant risk factors for maternal outcome, while right ventricular upper and lower diameter and pulmonary artery width were not significant risk factors. The acceleration of tricuspid regurgitation suggests high pulmonary systolic pressure, and pulmonary hypertension is related to cardiac remodeling and the expansion of main pulmonary artery. Considering the strong collinearity between variables, only the most significant variables are retained in the multivariate regression. 
However, according to the results of multivariate regression analysis, it was found that the severity of pulmonary hypertension, the increase of right heart structure and the deterioration of cardiac function were the risk factors of poor maternal outcome.

The blood volume of women of childbearing age increases gradually from 6-8 weeks of pregnancy. The cardiovascular system is most significantly affected by the change of blood volume. In the middle and late pregnancy, the blood volume may increase by nearly $50 \%$ compared with the peak value in non-pregnant patient. At the end of pregnancy, the maternal blood volume will increase by about $40-100 \%$ compared with the baseline level [10]. In this process, due to the increase of blood volume, the return blood volume of the right cardiac system will increase accordingly, and each cardiac cavity will also have mild expansion and hyperplasia. In addition, sex hormones have the effect of positive muscle strength, which can enhance myocardial contractility. Therefore, under normal circumstances, cardiac output can also increase in the same proportion; Under normal circumstances, in order to meet the demand for cardiac output during pregnancy, systemic vasodilation (including pulmonary artery) leads to a corresponding decrease in vascular resistance. Therefore, there is no significant change in maternal pulmonary artery pressure [11]. Normal pulmonary vessels can accommodate increased blood volume associated with pregnancy without significant cardiopulmonary damage [12].

However, in patients with pulmonary hypertension, the adaptability of the right ventricle to pressure load determines the functional status and prognosis of patients with pulmonary hypertension. Studies have confirmed that right ventricular dilation or left ventricular contraction can independently predict the low survival rate of patients with pulmonary hypertension. However, the enlargement and expansion of the right ventricle still play a key role. Some data show that the acute mortality of adult patients with pulmonary hypertension and right ventricular failure is $41 \%$, which is far higher than that of patients with left ventricular failure [13]. In addition, the risk of myocardial ischemia in patients with pulmonary hypertension will also increase, mainly due to the decrease of right coronary artery perfusion pressure and the asynchronous remodeling and extension of coronary artery and myocardium. Therefore, right ventricular ischemia is also common [14]. In addition, during delivery, uterine contraction will lead to the automatic infusion of 300-500 $\mathrm{ml}$ of blood in the uterus into the maternal circulation, resulting in increased preload of the right ventricle and pulmonary vascular bed. In addition, venous reflux may increase due to the remission of Inferior 
uterine vein obstruction. The resulting increase in cardiac output may last more than 48 hours, resulting in an increased risk of adverse maternal outcomes [15]. According to the results of this study, relevant literature and the research results of other scholars, it is considered that the severity of pulmonary hypertension and the abnormality of right heart function or structure may be the factors affecting maternal outcome. The mechanism may be that in patients with pulmonary hypertension, pulmonary vascular remodeling and the increase of pulmonary artery resistance limit the ability of the heart to process blood volume and compensatory increase cardiac output, resulting in right ventricular failure. With the decline of right ventricular function, or asynchronous ventricular contraction, and the decrease of right ventricular stroke volume, the left ventricular return blood volume will be reduced, resulting in the decrease of left ventricular preload. The decrease of left ventricular filling may also be due to the strong compliance of the right ventricle compared with the left ventricle. Therefore, due to the extension of systolic time and the excessive increase of the right heart, the ventricular septum bends to the left, resulting in the decrease of left ventricular volume in the early diastole. Coupled with right ventricular systolic and diastolic dysfunction, cardiac output decreased [16]. Therefore, monitoring the right heart structure of pregnant women may be helpful to predict maternal outcomes.

4.2 Effect of pulmonary hypertension and right heart enlargement on neonatal outcome

In this study, through data analysis, it was found that in univariate analysis, tricuspid regurgitation velocity and structural changes of right heart were significant risk factors for neonatal outcome. However, in multivariate analysis, only tricuspid regurgitation velocity and upper and lower diameter of right atrium were significant risk factors, while the diameters of right ventricle and pulmonary artery were not significant.

The development and maintenance of neonatal homeostasis mainly depend on the role of placenta. At present, studies have proved that the level of intrauterine oxygen concentration and the maintenance of oxygen homeostasis are involved in regulating placental formation, affecting placental structure and function, and further affecting fetal growth and development [17]. Although it is difficult to quantify the degree and intensity of hypoxia, let alone detect its occurrence, through a large number of clinical and basic studies, it has been generally accepted that fetal dysplasia is often related to placental hypoxia [18]. Fetal growth restriction is a common disease derived from 
early placental developmental defects, so most adverse pregnancy outcomes can be traced to placental developmental defects or dysfunction [19]. Current studies suggest that the etiology of placental developmental defects may be poor trophoblast invasion. During normal placental development, oxygen supply is one of the key regulatory factors affecting trophoblast differentiation, proliferation and migration. Therefore, hypoxia is likely to be the key factor leading to poor invasion of placental trophoblast [20].

In addition to the effects caused by hypoxia, some studies have found that venous congestion may also hinder the invasion of trophoblast in early pregnancy, resulting in abnormal placental function and development [21]. Right ventricular dysfunction is one of the mechanisms leading to changes in venous pressure and venous congestion. In patients with pulmonary hypertension, it often leads to increased afterload of the right heart. However, under normal circumstances, the right ventricle can increase myocardial contractility through centripetal remodeling, so as to adapt to the increase of cardiac load and ensure the normal cardiac output of patients. Continuously elevated pulmonary artery pressure can promote the parallel connection of myocardial sarcomeres and cardiomyocyte hypertrophy, resulting in the increase of right ventricular muscle mass and finally adaptive hypertrophy. However, adaptive hypertrophy is limited. When pulmonary artery pressure or right ventricular pressure is out of control, it will lead to RV-PA "uncoupling", that is, the right ventricle can no longer respond to changes in afterload. The right ventricle began to turn and expand to comply with the wall tension, resulting in a mismatch between oxygen supply and demand [22]. Therefore, in theory, when the patient's pulmonary hypertension progresses to a certain extent, especially when severe diastolic heart failure occurs, in order to compensatory increase the cardiac compliance, the patient's right heart begins to promote the series of cardiomyocytes and lead to the increase of myocardial eccentricity. The result will be the increase of the patient's cardiac cavity diameter. Therefore, it is common to increase the central cavity diameter in patients with severe pulmonary hypertension and right ventricular dysfunction. Combined with the review of the above literature and related studies, it is considered that the severity of pulmonary hypertension and the abnormal structure of the right cardiac system may be the risk factors of adverse neonatal outcomes. The mechanism may be the remodeling of the right cardiac system caused by pulmonary hypertension, resulting in a series of subsequent hypoxia and venous congestion. Therefore, the ultrasonic index of right heart may be applied to predict the outcome of newborns to a certain extent 
in practical clinical work.

\section{Conclusion}

In this study, we analyzed the clinical data of patients with pregnancy complicated with pulmonary hypertension in our hospital in recent years, and we found the changes of right heart structure (the acceleration of tricuspid regurgitation velocity, the increase of diameter of right atrium, right ventricle, right ventricular outflow tract and main pulmonary artery), cardiac function are important factors affecting the pregnancy outcome of pregnant women with pulmonary hypertension. In addition, we preliminarily designed a risk score through multifactor analysis, which is helpful to predict the possible outcome of puerperal period and neonatal outcome, hoping to be further improved in the future, and provide some reference for clinicians and patients to decide whether to continue pregnancy in practical work.

Pulmonary hypertension is still a very dangerous factor for pregnancy. Clinicians need to fully evaluate the right cardiac function and structure of patients when making decisions, and to analyze them according to the actual situation of patients. An appropriate therapy should be carefully selected to intervene pulmonary hypertension during pregnancy due to different disease degrees and clinical characteristics.

\section{Declarations}

Ethics approval and consent to participate: This study was carried out in accordance with the recommendations of Guangdong General Hospital, Guangdong Academy of Medical Sciences. The protocol was approved by the Guangdong General Hospital, Guangdong Academy of Medical Sciences. We have explained the details of this trial to the patient, including their rights, potential benefits and risks, and given them a signed copy of the informed consent. And we have got a consent statement from each subject.

Consent for Publication: The results, data and tables in this manuscript have not been published elsewhere, nor are they under consideration by another publisher.

Availability of data and materials: The raw data supporting the conclusions of this manuscript will be made available by the authors, without undue reservation, to any qualified researcher.

Competing interests: The authors declare that the research was conducted in the absence of any 
commercial or financial relationships that could be construed as a potential conflict of interest.

Funding: No individual or institution has funded our research

Author Contributions: Kaixun Zhao is the first author of this study. He is mainly responsible for the preliminary literature search and the design of the research process. In addition, data collection and article writing are also completed by this author. Professor Caojin Zhang is responsible for the direction of the research and provides professional advice and guidance to the authors. Ziyang Yang is responsible for the selection of statistical methods. Yin Zhou and Nanshan Xie mainly assisted in data analysis and table design. Dr. Disheng Lai reviews manuscripts and helps correct grammatical and methodological errors.

Acknowledgments: We are very grateful to Guangdong Provincial People's Hospital and Guangdong Academy of Medical Sciences for providing me with a large number of data and samples. Thanks to professor Caojin Zhang for his patient instruction and the help of all the researchers.

\section{Amendment}

This study was carried out in accordance with the recommendations of Guangdong General Hospital, Guangdong Academy of Medical Sciences. The protocol was approved by the Guangdong General Hospital, Guangdong Academy of Medical Sciences. The raw data supporting the conclusions of this manuscript will be made available by the authors, without undue reservation, to any qualified researcher.

This study is a retrospective study, and there is no human experiment or human tissue experiment. At present, it has been approved by our hospital and Research Institute. Our research data are from patients admitted to our hospital in recent years, and we have obtained the consent of all patients and all methods were carried out in accordance with relevant guidelines and regulations

\section{Consent statement of subjects}

I have read the above introduction of this study, and I am glad to have the opportunity to discuss with doctors and ask questions about this study. All my questions have been answered satisfactorily. I am aware of the risks and benefits of participating in this study. I am aware that participating in the study is voluntary and I have confirmed that there is sufficient time to consider this and 
understand:

. I can always ask my doctor for more information.

- I can withdraw from this study at any time without discrimination or retaliation, and medical treatment and rights will not be affected.

I also know that if I withdraw from the study halfway, especially when I withdraw from the study due to treatment reasons, it will still be very beneficial for me and the whole study if I tell the doctor about the change of my condition and complete the corresponding physical and chemical examination.

If I need to take any other treatment due to the change of my condition, I will consult the doctor in advance or tell the doctor truthfully afterwards.

I agree that the drug regulatory authority, ethics committee or the representative of the implementer can refer to my research materials.

I agree to use my medical records and pathological examination specimens for other studies other than this study.

I will be provided with a signed and dated copy of the informed consent.

Finally, I decided to agree to participate in the study and follow the doctor's orders as much as possible.

\section{Reference}

[1] Condliffe, R. , et al. "Connective tissue disease-associated pulmonary arterial hypertension in the modern treatment era. " American Journal of Respiratory \& Critical Care

Medicine 179.2(2009):151.

[2] Kane, Mc Goon, Garvan C . "Pulmonary Hypertension: Diagnosis and Management." Mayo Clinic Proceedings 84.2(2009):191-207.

[3] Lisa, et al. "Pulmonary hypertension: types and treatments. " Current Cardiology Reviews (2015).

[4] Lai, Y. C. , et al. "Pulmonary Arterial Hypertension: The Clinical Syndrome." Circulation Research 115.1(2014):115-130.

[5] Anna, et al. "Statement on Pregnancy in Pulmonary Hypertension from the Pulmonary Vascular Research Institute." Pulmonary Circulation (2016).

[6] Barbagallo, M. , et al. "Vascular Effects of Progesterone : Role of Cellular Calcium Regulation. " Hypertension 37.1(2001):142.

[7] Olsson, K. M. , and X. Jais . "Birth Control and Pregnancy Management in Pulmonary Hypertension." Seminars in Respiratory \& Critical Care Medicine 34.05(2013):681-688. 
[8] Elisabeth Bédard,, Konstantinos Dimopoulos, and Michael A. Gatzoulis,, *. "Has there been any progress made on pregnancy outcomes among women with pulmonary arterial hypertension?." European Heart Journal 30.3(2009):256.

[9] Sun, Xuefeng , J. Feng , and J. Shi . "Pregnancy and pulmonary hypertension: An exploratory analysis of risk factors and outcomes." Medicine 97.44(2018):e13035.

[10] Soma-Pillay, P. , et al. "Physiological changes in pregnancy." Cardiovascular Journal of Africa 27.2(2016):89-94.

[11] Hall, Michael E et al. "El corazón durante el embarazo" [The heart during pregnancy]. Revista espanola de cardiologia vol. 64,11 (2011): 1045-50.

doi:10.1016/j.recesp.2011.07.009

[12] Safdar, and Z. "Pulmonary arterial hypertension in pregnant women." Therapeutic Advances in Respiratory Disease 7.1(2013):51.

[13] Chaouat, A. , et al. "Role for interleukin-6 in COPD-related pulmonary hypertension.

" Chest 136.3(2009):678-687.

[14] Thenappan, Thenappan, et al. "Pulmonary arterial hypertension: pathogenesis and clinical management." Bmj (2018):j5492.

[15] Madden, and P. B. . "Pulmonary Hypertension and Pregnancy." Obstetric Anesthesia Digest 30.1(2010):9-11.

[16] Aguero, J. , et al. "Characterization of right ventricular remodeling and failure in a chronic pulmonary hypertension model." AJP Heart and Circulatory Physiology 307.8(2014):1204-15.

[17] BH Fryer, and MC Simon. "Perspective Hypoxia, HIF and the Placenta." Cell Cycle (2006).

[18] Rampersad, R. , and D. M. Nelson . "Trophoblast biology, responses to hypoxia and placental dysfunction in preeclampsia. " Front Biosci 12.1(2007):2447-2456.

[19] Fisher, S. J. . "The placental problem: Linking abnormal cytotrophoblast differentiation to the maternal symptoms of preeclampsia." Reproductive Biology and Endocrinology 2.1(2004):53.

[20] Genbacev, O. , et al. "Regulation of Human Placental Development by Oxygen Tension." Science 277.5332(1997):1669-1672.

[21] Gyselaers, W. , and L. Peeters . "Physiological implications of arteriovenous anastomoses and venous hemodynamic dysfunction in early gestational uterine circulation: a review." Journal of Maternal-Fetal Medicine 26.9(2013):841-846.

[22] A, Harm J. Bogaard, , et al. "The Right Ventricle Under Pressure." Chest 135.3(2009):794804. 